\title{
Compressed Lozenge Dose Form
}

National Cancer Institute

\section{Source}

National Cancer Institute. Compressed Lozenge Dose Form. NCI Thesaurus. Code C149374.

Solid single-dose preparation intended to be sucked to obtain a local or systemic effect.

It is prepared by compression and is often rhomboid in shape. Compressed lozenges usually contain flavoring and sweetening agents. They dissolve or disintegrate slowly when sucked. 